\title{
Comparison of Clutch Hysteresis Characteristics Depending on the Pedaling Time and the Compressed Air
}

\author{
Hyun-sik Kim ${ }^{1, a}$, Sung-mo Yang ${ }^{1, ~ b}$, Hyang-woo Kim ${ }^{2, \mathrm{c}}$ \\ ${ }^{1}$ Department of Mechanical System Engineering, Chonbuk National University, \\ Jeonju, 54896, South Korea \\ ${ }^{2}$ Department of Automotive Plant Industrial Engineering, Hanyeong College, \\ Yeosu, 59720, South Korea \\ ahyunsiclove@jbnu.ac.kr, ${ }^{b}$ yangsm@jbnu.ac.kr, chwkim4035@hanmail.net
}

\section{Keywords: Hysteresis, Clutch Booster, Pedaling Time, Compressed Air}

\begin{abstract}
In this paper, we compared, through experiments, the hysteresis characteristics in the load of the push rod, that is generated in the booster cylinder according to the time of the instantaneous actions made by the driver in stepping on and releasing the clutch pedal. In addition, we performed separated experiments to identify how the hysteresis characteristics of the pedal effort generated in the pedal unit and the hysteresis characteristics of the pedal effort that occurred in the booster push rod when the driver stepped on the clutch pedal, appeared in varied form, in a case when the compressed air of 8.0bar was applied into the internal booster and in another case without applying the compressed air. As the experimental results, it was found that when the compressed air was applied, the stroke of the push rod has increased and thereby the maximum load continued to be applied to the booster. Also it was confirmed that the load applied to the pedal unit when you step on the clutch pedal was found to be $25 \mathrm{kgf}$ when unapplied showing an increase of more than three times compared to $7.5 \mathrm{kgf}$ recorded when the compressed air was applied, which will accordingly cause an increase in degree of fatigue of the driver.
\end{abstract}

\section{Introduction}

The vehicle with a manual transmission employs a clutch system which is needed for applying the desired shift during power transmission. The clutch system is commonly widely used for the standard and large size commercial vehicles, despite the trend of decrease due to the recent increase of the automatic transmission vehicle. The clutch is subject to a lot of additional equipment which is mounted on the path from the clutch pedal for speed change to be managed by the driver to the clutch plate for final operation because the degree of the torque to be delivered is higher compared to passenger cars. Since such a clutch system in the whole makes the driver repeatedly step on the pedal, it presents a problem to cause the increased fatigue of the driver, so a hydraulic device for reducing the pedal unit strength are being applied in order to reduce such degree of fatigue of the driver and a variety of study to linearize the characteristics of the pedal effort on the clutch behavior mechanism has been proceeded [1].

Clutch system mainly consists of the unit comprising Clutch disk, Pressure plate, Clutch shaft, Clutch spring, and the parts of the hydraulic function such as Clutch oil lines, master cylinder, release fork, booster cylinder, Release bearing and so on. The following Fig. 1 is to show the appearance of a typical friction clutch and such clutches are divided into a friction clutch, a fluid clutch, and an electromagnetic clutch by the operation method.

In such hysteresis characteristics, it is expected that the force applied to the booster cylinder of the hydraulic pressure for driving the clutch system varies also according to the time of stepping on and off the clutch pedal by the driver. Accordingly we aim to test and analyze the characteristics in the pedal efforts variation of a booster cylinder according to the change by parameterizing the standard pedaling habit of the driver by the pedaling time. Also we have analyzed the hysteresis characteristics of the booster cylinder and the characteristics of the pedal efforts on the pedal unit 
according to whether the compressed air was applied to the internal booster or not. Typically, the stroke of the pedal unit against the clutch system and the hysteresis characteristics of the pedal efforts which represent the pressure force applied to the pedal unit shows the non-linear behavior as shown in Fig. 2, and confirms that the width of hysteresis and the magnitude of the pedal efforts vary according to a number of key variables in the clutch system. In such hysteresis characteristics, it is expected that the force applied to the booster cylinder of the hydraulic pressure for driving the clutch system varies also according to the time of stepping on and off the clutch pedal by the driver. Accordingly we aim to test and analyze the characteristics in the pedal efforts variation of a booster cylinder according to the change by parameterizing the standard pedaling habit of the driver by the pedaling time.

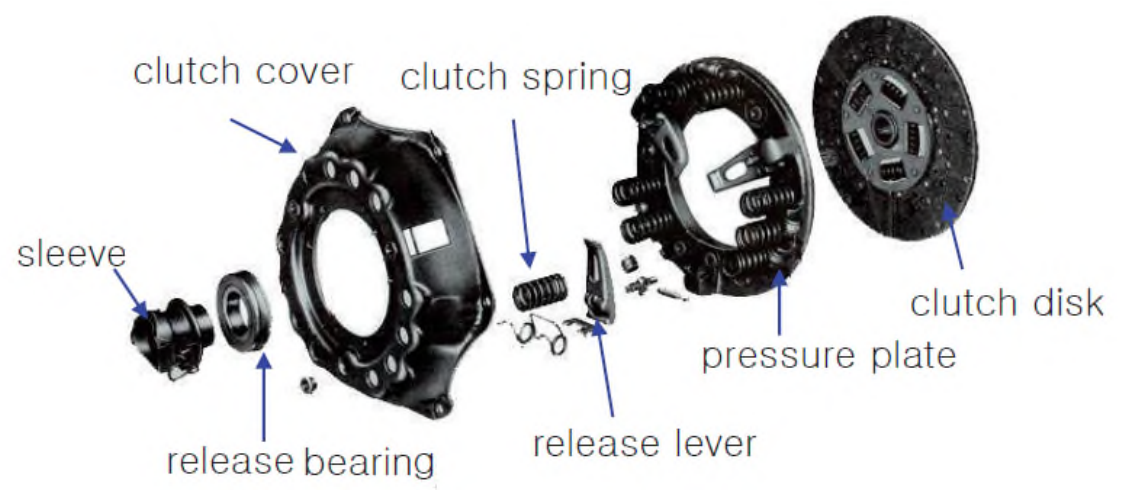

Fig. 1 Clutch system.

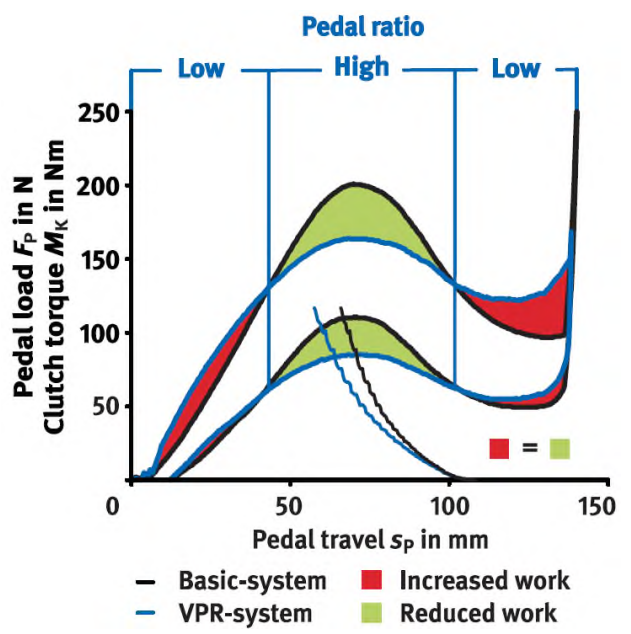

Fig. 2 Pedal load measurement with and without variable pedal ratio [2].

\section{Mathematical Modeling of Clutch Cylinder and Hydraulics Unit}

Where, in the context of the whole driving system, the power transmission system in the clutch system is viewed with a simplified mathematical model, then it is presented the same as in Fig. 3 . By means of the equalization setting of the parts which constitute the transmission system and also by connecting the equivalent components, those delivery systems were modeled in three variables such as mass, rigidity and damping. Equilibrium equation of the forces on the internal pressure of the hydraulic piston of the clutch cylinder for this modeling, is the same as shown in the following formula [2].

Here are $P_{c}$ is inner line pressure, $X_{m}$ and $X_{r}$ is cylinder displacement, $X_{p}$ is push rod displacement, $\beta$ is bulk modulus, $V$ is oil pressure volume, $M_{m}$ and $M_{r}$ is piston mass, $b_{m}$ and $b_{r}$ is viscosity coefficient, $k_{m}$ and $k_{r}$ is return spring invariable, $k_{p}$ is push rod spring invariable, $A_{m}$ and $A_{r}$ is piston area, $f_{m}=k_{p}\left(X_{p}-X_{m}\right)$ is load spring capacity and $f_{d}=k_{l}\left(X_{l}-X_{r}\right)$ is load spring capacity. The interaction formula of these mathematical modeling are as follows. 


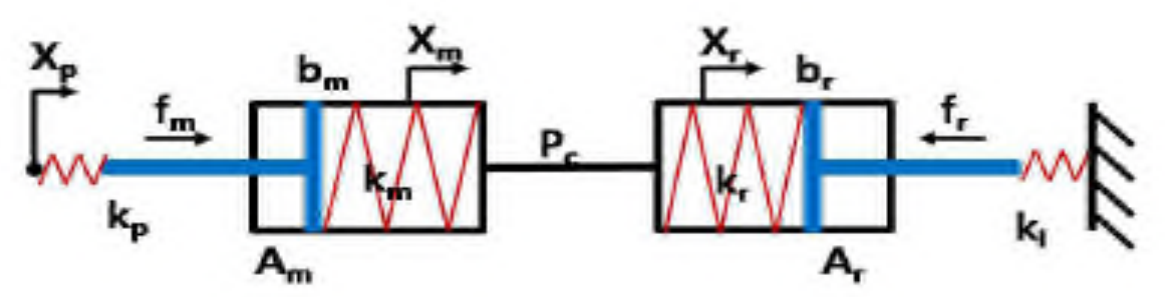

Fig. 3 A schematic of clutch hydraulic system.

$\dot{P}_{\sigma}=\frac{\hat{\beta}}{V}\left(A_{m} \dot{x}_{m}-A_{r} \dot{x}_{r}\right)$

$M_{m} \ddot{x}_{m}+b_{m} \dot{x}_{m}+k_{m} x_{m}=f_{m}-A_{m} P_{g}$

$=k_{p} x_{p}-\left(A_{11} x_{m}-A_{12} x_{r}\right)$

$M_{r} \ddot{x}_{r}+b_{r} \dot{x}_{r}+k_{r} x_{r}=A_{r} P_{q}-f_{l}$

$$
=\left(A_{12} x_{m}-A_{22} x_{r}\right)-f_{l}
$$

$A_{11}=A_{m} A_{m}$

$A_{12}=A_{m} A_{r}$

In this formula, the non-linear equations of motion was set up for an experiment in the study and, in order to identify the parameter to interpret it, the variables for each single item were measured and their values were used as the variables while the values of the initial pedal moving were added with the sin function value. Considering that the equation is complex and calculations take a long time since both the property value of the diaphragm and the value of the pedal return spring have the non-linear values, the non-linear expression was substituted after initially the verification of the entire system has been approximated in a linear expression form. The parameters of the master cylinders and the clutch boosters used in this experiment are the same as shown in the following Table 1.

Table 1. Specifications of master cylinder and clutch booster.

\begin{tabular}{|c|c|c|}
\hline Properties & Master Cylinder & Clutch Booster \\
\hline Mass & $27.56 \mathrm{~g}$ & $628.76 \mathrm{~g}$ \\
\hline $\begin{array}{c}\text { Diameter } \\
\text { (Inside, Outside) }\end{array}$ & $26 \mathrm{~mm}, 35 \mathrm{~mm}$ & $25 \mathrm{~mm}, 80 \mathrm{~mm}$ \\
\hline Area & $5.31 \times 10^{4} \mathrm{~m}^{2}$ & $4.91 \times 10^{4} \mathrm{~m}^{2}$ \\
\hline Stiffness & $3,000 \mathrm{~N} / \mathrm{m}$ & $1,500 \mathrm{~N} / \mathrm{m}$ \\
\hline
\end{tabular}

Based on the above experimental results, we aim to perform the experiment to identify the hysteresis characteristics of the pedal efforts on the clutch booster cylinder, to be varied due to the speed variation of the instantaneous actions of stepping on and off the pedal, and the experiment of the hysteresis characteristics of the booster cylinder tested with and without the compressed air applied to the internal booster as well as the test on the characteristics of the pedal efforts of the pedal unit.

\section{Hysteresis Property Test According to the Time Variation of Pedaling}

The experiment was performed with the booster cylinder applied with pressed air, setting the time rang of the pedal stepping from the minimum of $0.6 \mathrm{sec}$ to the maximum $4.0 \mathrm{sec}$ while the time range for releasing the pedal from the minimum $0.7 \mathrm{sec}$ to the maximum $3.8 \mathrm{sec}$. The experiment was not able to measure the time variation of pedal stepping completely proportionally because measurement should be assessed in a very short time. The measured result is shown in Fig. 4. 


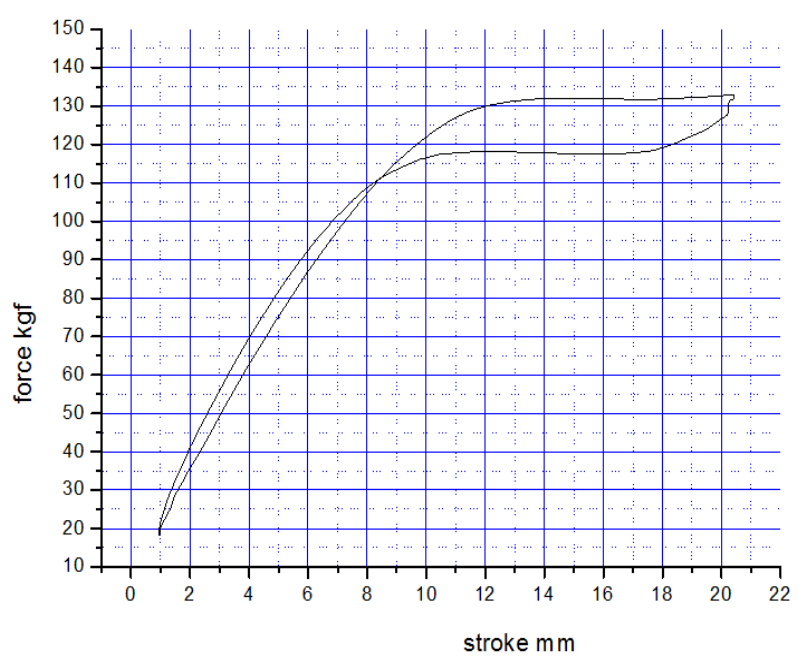

(a) Push time $0.6 \mathrm{sec}$ vs releasing time $0.7 \mathrm{sec}$.

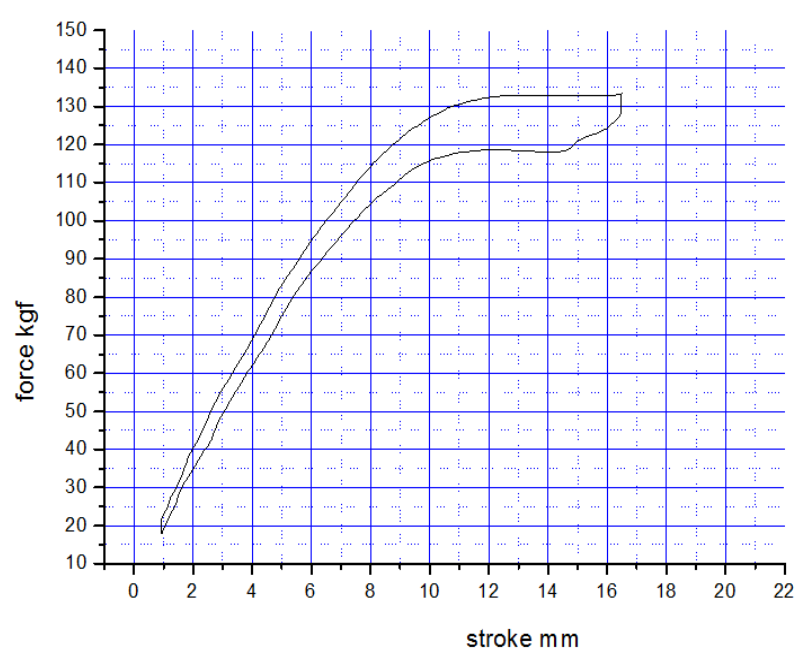

(c) Push time $2.3 \mathrm{sec}$ vs releasing time $2.3 \mathrm{sec}$.

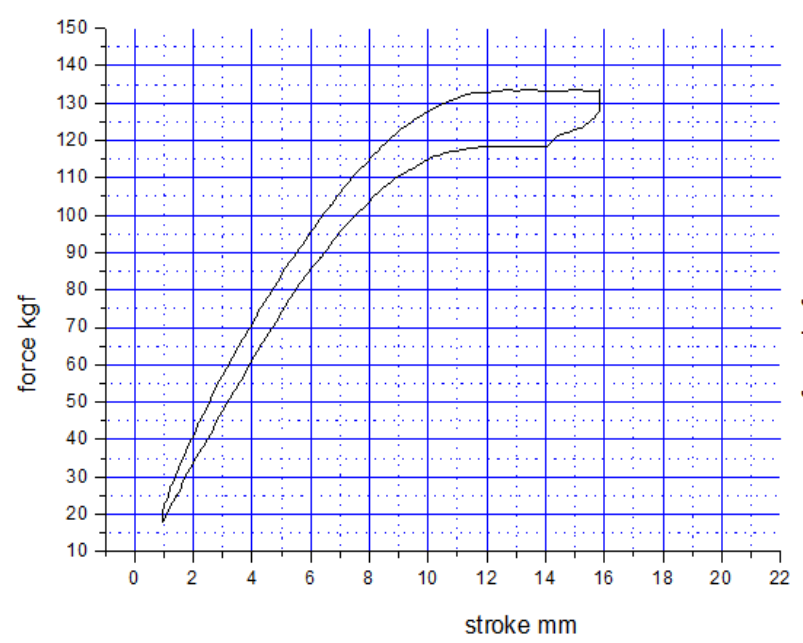

(e) Push time $4.0 \mathrm{sec}$ vs releasing time $3.5 \mathrm{sec}$.

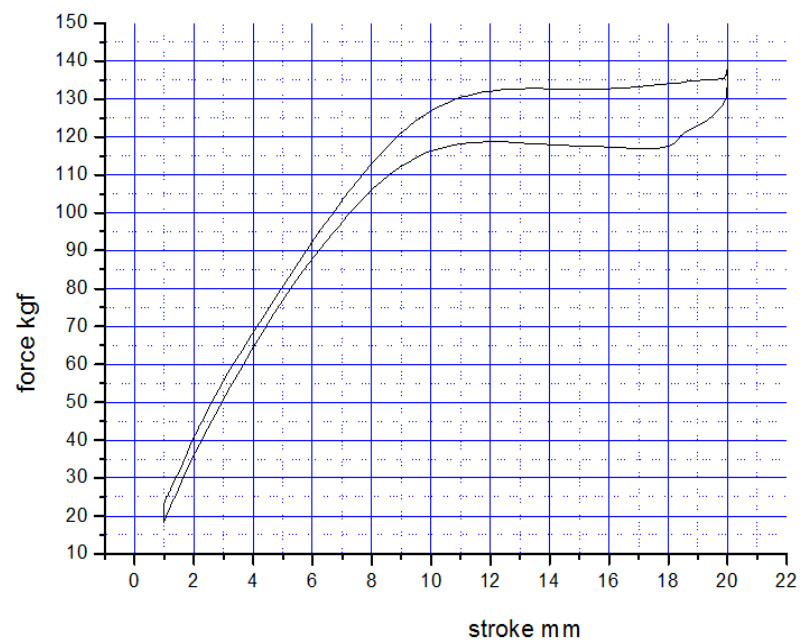

(b) Push time $1.7 \mathrm{sec}$ vs releasing time $1.7 \mathrm{sec}$.

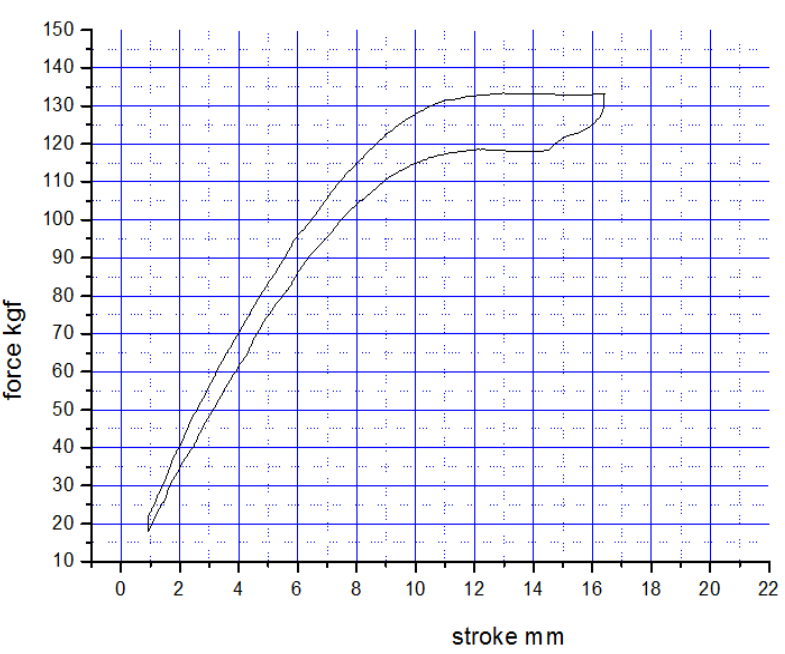

(d) Push time 3.0sec vs releasing time 3.3sec.

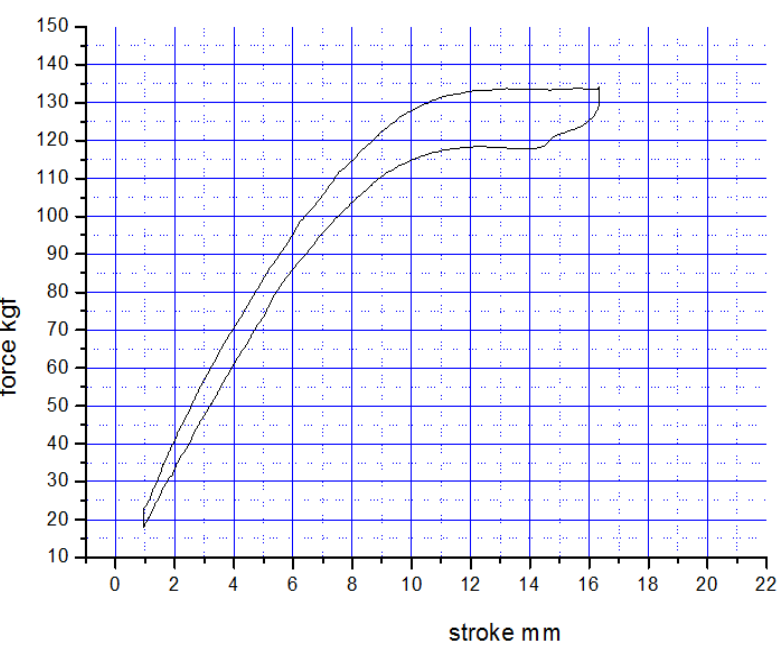

(f) Push time $4.0 \mathrm{sec}$ vs releasing time $3.8 \mathrm{sec}$.

Fig. 4 Graph of the results of hysteresis of the booster push rod due to time-variation of pedaling.

The experimental results confirm that the booster cylinder stroke is reduced as the time at which the driver step on and off the pedal is increased and accordingly the maximum load displacement is getting shorter from $14 \mathrm{~mm}$ to $12 \mathrm{~mm}$. In addition, the width of the load change of the hysteresis loop grows, and this kind of the hysteresis characteristics may produce disadvantage in the clutch actuation mechanism. 


\section{Hysteresis Characteristics Tests with the Compressed Air}

Next, experiments was carried out with respect to the hysteresis characteristics of the pedal efforts on the pedal unit and of the load applied to the booster push rod with the compressed air applied to the booster cylinder or without that. This experiment was carried out with oil lines of $2 \mathrm{~m}$ length and OD $8 \mathrm{~mm}$, made of steel material and the time to stepping on and off the pedal was set as 2sec. The test results are the same as in the following Fig. 5.

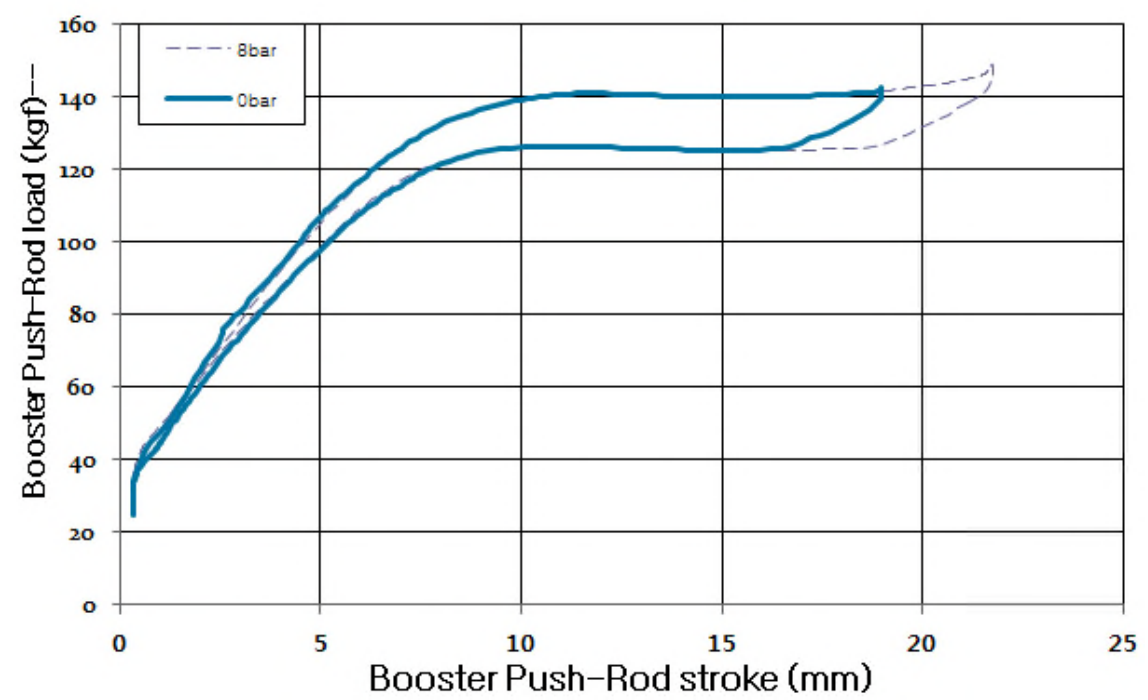

Fig. 5 Graph of the hysteresis test results of the booster cylinder with the compressed air applied and the case without that.

In addition, the resulting pedal stroke and the hysteresis characteristics of the load applied to the pedal, on which the driver is to step, compared with the hysteresis characteristics to the clutch booster are as follows as shown in Fig. 6.

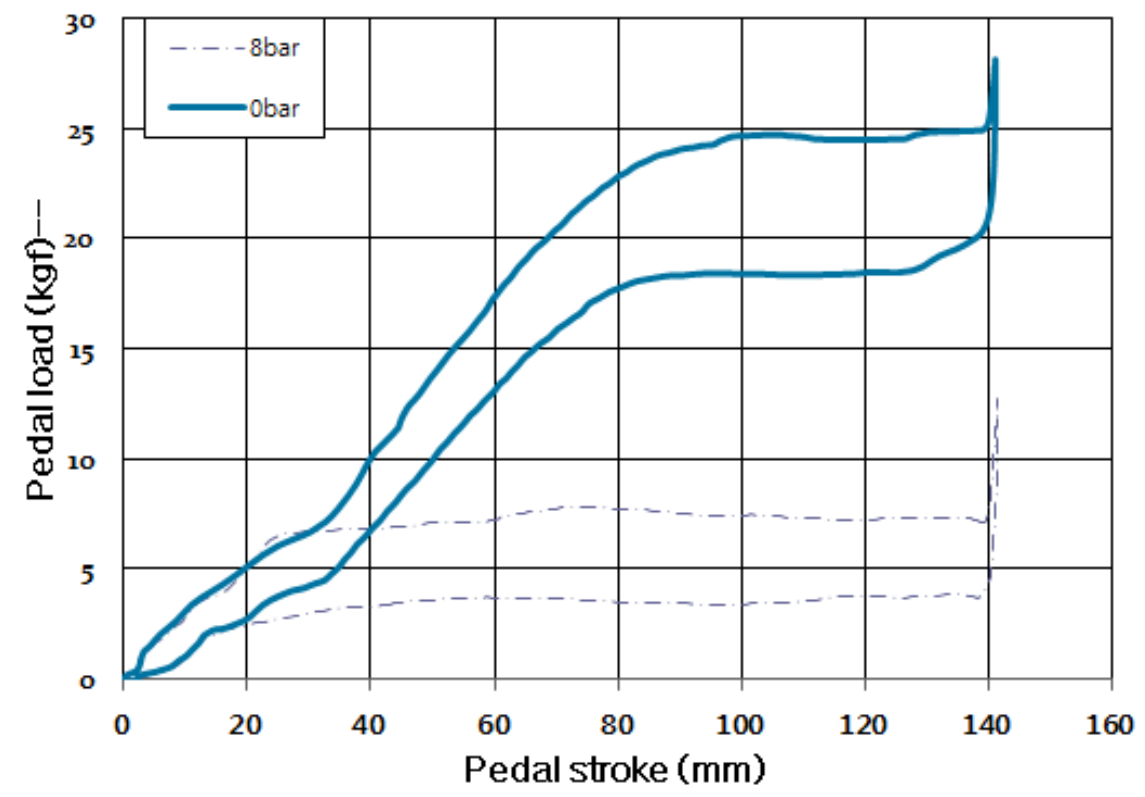

Fig. 6 Graph of the hysteresis test results of the pedal efforts according to the presence or absence of the compressed air applied.

The results of this experiment confirmed that the stroke caused in the booster decreased with the compressed air unapplied and instead the load applied to the clutch pedal unit tended to noticeably increase. The increasing load that occurred in the section of clutch pedal imply that the load 
resulting from the pedal stepping by the driver increased, so it is considered that the fatigue of the operator may decreases when the pressured air is applied.

\section{Conclusion}

In this paper, we compared the hysteresis characteristics of the booster cylinder and the pedal efforts in the pedal parts in the clutch pedal system for the manual transmission.

By setting the time to be taken by the actual driver for stepping on and off the clutch pedal as a variable, we have compared the hysteresis characteristics of the pedal efforts caused in the booster cylinder and further compared the hysteresis characteristics for the booster push rod as well as the pedal efforts in the pedal unit according to the cases of the presence and absence of the compressed air that was applied to the booster cylinder.

Experimental results confirmed that the longer the time of the instantaneous pedaling takes, the more the stroke in the booster push rod decreases while the hysteresis width of the pedal efforts to the corresponding part is reduced. Further, the stroke of the push rod is increased when the compressed air is applied to the internal booster, thereby it can be seen that the load for driving the clutch system continues. In addition, the load applied to the pedal unit when the driver actually steps on the clutch pedal is decreased to $30 \%$ or less compared than the case when the pressed air is not applied, so it is considered that the fatigue of the driver can be lowered due to this effect.

\section{Acknowledgement}

This work was supported by the National Research Foundation of Korea(NRF) Grant funded by the Korean Government(MOE)(No. NRF-2011-0010475)

\section{References}

[1] I. Kenji, Y. Masatoshi, Pressure Pulsation Transfer in Clutch Hydraulic System, Toyota central Research \& Development Labs.

[2] H. Tankahisa, Y. Hiroshi, Experimental Study of Reduction Methods for clutch Pedal Vibration and Drive Train Rattling Noise from Clutch System, Soc. Automot. Eng. 932007, 39-44 